\title{
La proyección fría (CGS): Una alternativa a las tecnologías convencionales de deposición ${ }^{(*)}$
}

\author{
M. Villa*, S.Dosta*, J. Fernández* y J. M. Guilemany*
}

\begin{abstract}
Resumen
La proyección fría es una tecnología novedosa para la obtención de recubrimientos y recargues, que se incluye en el campo de la proyección térmica. Debido a que las temperaturas de trabajo son relativamente bajas (siempre por debajo de los puntos de fusión del material), es una técnica ideal para depositar materiales sensibles a la temperatura como materiales nanoestructurados, amorfos o reactivos al oxígeno.La reducción en los costes (materia prima, gases...), la disminución de los tratamientos superficiales (pre-proyección como post-proyección), el aumento de la vida útil de las piezas en servicio, la disminución de los problemas asociados a la fusión del material proyectado (oxidación, corrosión, tensiones residuales a tracción...), el mantenimiento de las estructuras de partida, etc., han hecho de la proyección fría una técnica de proyección eficiente, más ecológica y económicamente más asequible que las técnicas de proyección y de deposición convencionales.
\end{abstract}

Palabras clave

Proyección fría; Estado del arte; Recubrimientos.

\section{Cold gas spray (CGS): An alternative to conventional deposition technologies}

Abstract

Keywords

\begin{abstract}
Cold spraying is a new technology to produce coatings and recharges that belongs to the field of thermal spraying. Since work temperature is relatively low (always lower than melting temperature), it is ideally for depositing temperaturesensitive materials such as nanophased and amorphous materials or oxygen-sensitive materials. Material cost reduction (raw materials, gases ...), reduction of surface treatments (pre- and post-spraying), possibility of increasing the pieces life, reduction of the problems associated with material melting (oxidation, corrosion, residual tensile stress ...), maintenance of the starting microstructures, etc., have made that cold spray technique an efficient, more ecological and economically more affordable than conventional spraying and deposition techniques.
\end{abstract}

Cold gas spray; Review; Coatings.

\section{INTRODUCCIÓN}

La proyección térmica consiste en dotar a un material o una mezcla de varios materiales de energía térmica y cinética para crear una fase líquida, o semilíquida. Las partículas en estas fases son proyectadas sobre una superficie gracias a un gas supersónico. Al impactar sobre la superficie, el material se extiende y rápidamente se solidifica formando la unidad básica microestructural de la deposición por proyección denominada "splat".

Los sistemas de proyección térmica pueden clasificarse en función de cómo se realiza el aporte térmico y cinético. Este aporte puede ser por corriente eléctrica o por combustión. En la figura 1 puede verse una comparativa de las diferentes técnicas de proyección:

Las limitaciones existentes en los procesos de proyección térmica (claves para el uso de nuevos materiales así como para la mejora en las aplicaciones de materiales comunes), en unas industrias cada vez más especializadas y exigentes, han generado el desarrollo de técnicas de proyección donde los costes y las eficiencias en la deposición adquieren un sentido imprescindible en el momento de elección de una técnica de recubrimiento.

La reducción en los costes de material (materia prima, gases...), la disminución de los tratamientos superficiales (pre-proyección como post-proyección), la posibilidad de aumentar la vida útil de las piezas en servicio, la disminución de los problemas asociados a

(•) Trabajo recibido el día 11 de febrero de 2011 y aceptado en su forma final el día 11 de noviembre de 2011.

* Centro de Proyección Térmica (CPT), Universidad de Barcelona. Martí i Franqués 1, Planta 1. Edificio facultad de Química. 08028

Barcelona. E-mail: mvilla@cptub.org. 


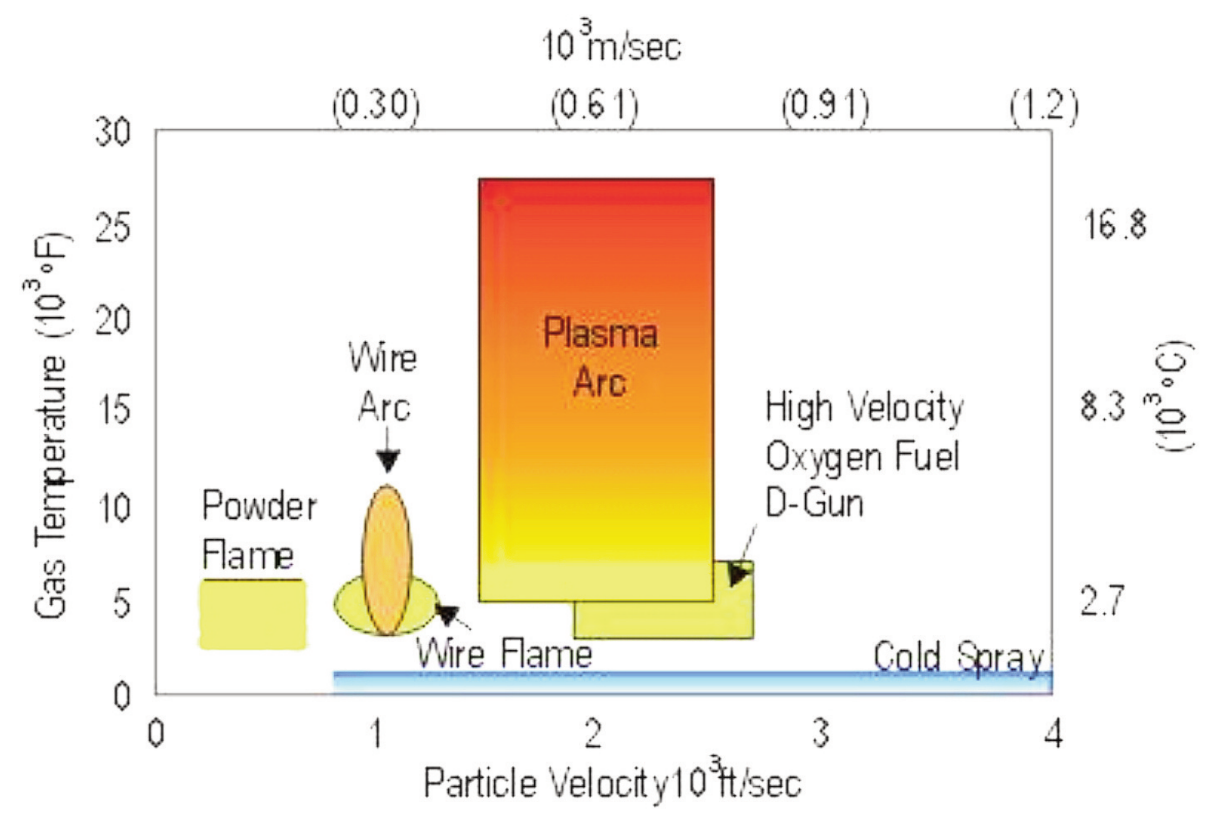

Figura 1. Comparativa de técnicas de proyección.

Figure 1. Spraying techniques comparative.

la fusión del material proyectado (oxidación, corrosión, tensiones residuales a tracción...), el mantenimiento de las estructuras de partida, etc., han hecho de la proyección fría una técnica de proyección eficiente, más ecológica y económicamente más asequible que las técnicas de proyección convencionales ${ }^{[1 \text { y } 2]}$.

La proyección fría es una técnica que no se limita únicamente a industrias con grandes presupuestos como la militar o la aeronáutica, sino que debido a su asequibilidad y a la amplia gama de materiales que pueden ser usados, ha permitido su aplicación en industrias como la del transporte o la de servicios.

Uno de los mayores atractivos de la técnica de proyección fría es la posibilidad de obtener recubrimientos con unas propiedades únicas, las cuales no son alcanzables a través de técnicas convencionales de proyección térmica. Esto es debido al hecho de que la deposición de los materiales sobre los sustratos para formar el recubrimiento tiene lugar en estado sólido. No se expone a los materiales a altas temperaturas de modo que estos materiales no alcanzan su temperatura de fusión. Debido a ello, reacciones no deseables de descomposición u oxidación, son prácticamente eliminadas ${ }^{[3}$ y 4$]$.

El objetivo principal de la técnica, así como todas las técnicas de proyección térmica, es la obtención de recubrimientos que mejoren, a nivel superficial, una o varias propiedades determinantes para la aplicación deseada. La eficiencia en la deposición, la adhesión y la estructura final del material proyectado dependen de numerosos factores, los cuales, al mismo tiempo dependen del tipo de material proyectado y del material sobre el que se proyecta.

\section{LA PROYECCIÓN FRÍA (COLD GAS SPRAY - C.G.S.)}

La proyección fría es un proceso de obtención de recubrimientos y recargues donde partículas de pequeño tamaño $(5-50 \mu \mathrm{m})$ son aceleradas por un gas presurizado (nitrógeno o helio) que se calienta a temperaturas comprendidas entre los 300 y los $800^{\circ} \mathrm{C}$, hasta alcanzar velocidades supersónicas. El mecanismo de este proceso está basado en las propiedades que adquieren las partículas al proyectarlas a una determinada presión y temperatura. El estudio y control de estas variables permite la obtención de recubrimientos a partir de partículas en estado sólido.

Hay una diferencia básica entre las técnicas convencionales de proyección térmica y la denominada proyección fría. Mientras que en las primeras el sistema necesita elevada energía térmica y cinética para formar el recubrimiento, en el caso de la proyección fría la energía cinética es mayoritaria en el proceso como puede observarse en la figura 2 .

Las principales ventajas que el proceso conlleva $\operatorname{son}^{[5-12]}$ :

- Disminución de la porosidad en los recubrimientos. El efecto compresivo de las partículas 


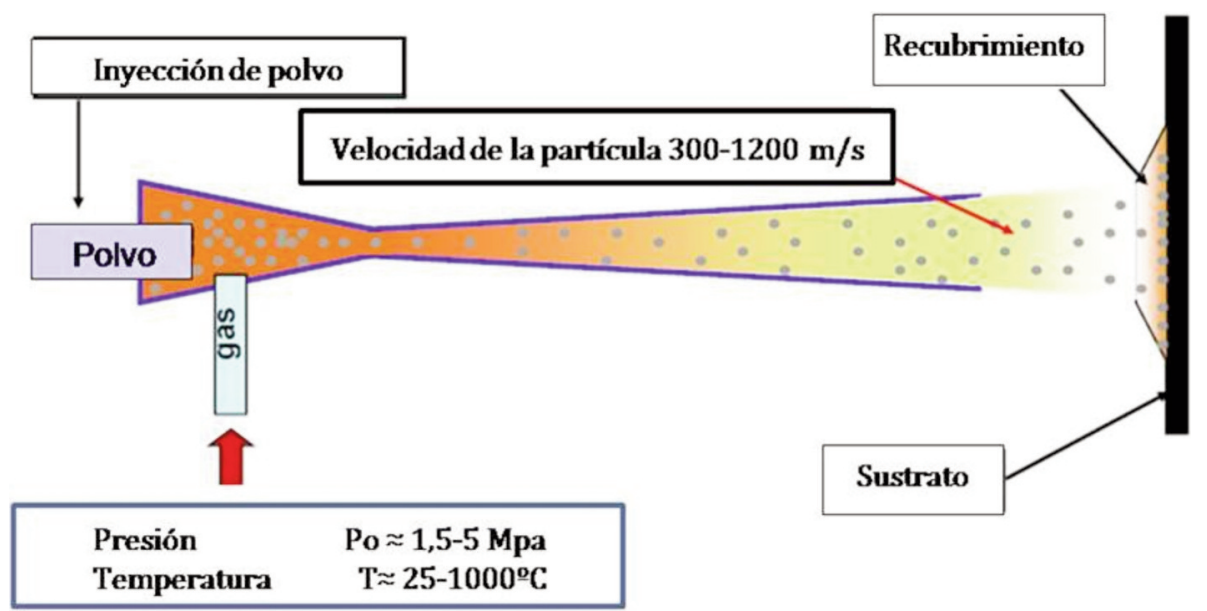

Figura 2. Esquema del sistema de proyección fría.

Figure 2. Cold spray system scheme.

que llegan a alta velocidad al sustrato, tiende a cerrar los pequeños poros y huecos existentes en las capas subyacentes de material.

- Alta eficiencia en la deposición (60-95\%) con capacidad para obtener recubrimientos de alta densidad y dureza.

- Disminución de óxidos en el recubrimiento. Al no fundir las partículas, la reactividad superficial de las mismas es menor que en el caso de las técnicas convencionales.

- Debido principalmente a que las partículas no se encuentran en estado fundido cuando llegan al sustrato, la alta presión aplicada hace que las tensiones residuales a tracción existentes en los recubrimientos generados por proyección térmica convencional, sean sustituidas en su mayoría por tensiones de tipo compresivo.

- La composición y la microestructura de los materiales de partida se mantiene.

- Mínima preparación del sustrato.

- Capacidad de obtener recubrimientos con alta conductividad eléctrica/térmica.

- Capacidad de proyectar materiales especiales termosensibles.

- Posibilidad de reutilización de las partículas no adheridas al sustrato (100\% de reciclaje).

- Incremento de la seguridad operacional debido a la ausencia de un haz de gas de alta temperatura, combustión, radiación o gases explosivos.

Si se comparan las velocidades de las partículas con respecto a la temperatura en las diferentes técnicas de proyección, se obtiene el gráfico de la figura 3, donde se observa que con la técnica de proyección fría se obtienen mayores velocidades de partícula a menores temperaturas.

\subsection{Variables del proceso}

Las condiciones en las que se realiza la proyección varían en función del tipo de material proyectado, el sustrato, sus composiciones, sus propiedades físicoquímicas, etc. Estas condiciones determinan la calidad del recubrimiento. La calidad es fijada por las necesidades establecidas por la aplicación para la cual se desea generar el recubrimiento (nivel de porosidad, espesor del recubrimiento, adherencia recubrimientosustrato, microestructura...).

La unión de las partículas al sustrato está influenciada por diversos factores. Estos factores van desde los parámetros geométricos, como la superficie de contacto y su rugosidad, hasta los parámetros termo-mecánicos como la deformación plástica, la tensión de fluencia, la presión y la temperatura en la interface.

Las principales variables que se deben tener en cuenta a la hora de llevar a cabo una proyección $\operatorname{son}^{[13-18]}$ :

- Presión.

- Temperatura.

- Velocidad de la partícula.

- Distancia de proyección.

- Morfología del polvo de proyección.

- Tasa de alimentación del polvo de proyección.

- Ángulo de la pistola de proyección.

- Rugosidad superficial del sustrato. 


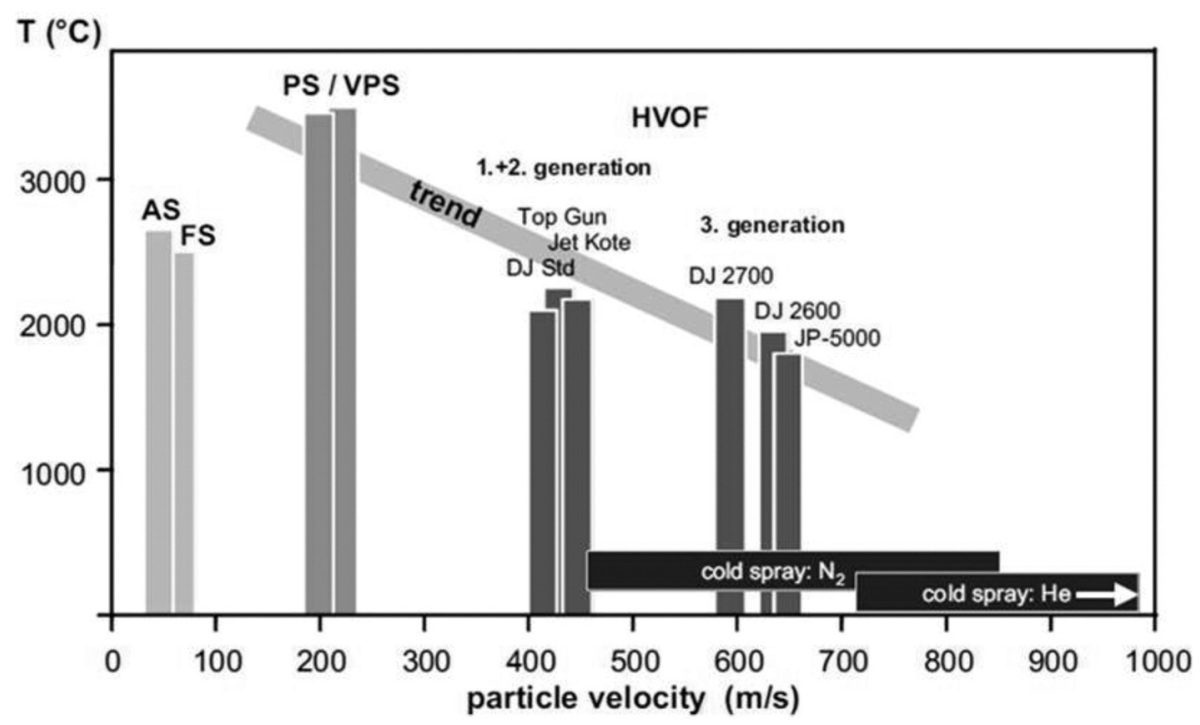

Figura 3. Gráfico de velocidad de partícula vs. temperatura.

Figure 3. Particle velocity vs. temperature graphic. AS-Arc Spray FS-Flame Spray PS/VPS-Plasma Spray/Vapour Plasma Spray HVOF-High Velocity Oxygen Fuel. The cold spray process and its potential for industrial applications F. Gartner et. al.

Además, deben tenerse en cuenta propiedades fisicoquímicas de los propios materiales como su dureza, plasticidad o reactividad superficial.

\subsection{Economía del proceso de proyección fría}

A parte de las ventajas tecnológicas, otra de las principales características de la técnica es la gran reducción de costes y plazos de ejecución. Para poder lograr estas disminuciones, es necesario tener en cuenta todos los aspectos involucrados en la fabricación específica de componentes. La mayor parte de las obras de ingeniería proceden de la fundición. Los costes implicados en los diferentes procesos de fabricación de una pieza de fundición se muestran en la figura 4 .

El estudio muestra que el proceso de fabricación usando la técnica de proyección fría incide simultáneamente en muchos aspectos de la cadena de valor. Este análisis de la cadena de valor muestra que una gran reducción de costes debe lograrse a través de la reducción de costes en todas las áreas de la cadena y no en una sola ${ }^{[19]}$.

El modelo de análisis de valor ha sido desarrollado por la empresa Pratt $\&$ Whitney como parte de un proyecto de deposición de polvo por láser usando titanio como materia prima. Este modelo de costes se aplicó a continuación al proceso de proyección fría de titanio. (i) Reducción en la entrada de material.

(ii) Eliminación del molde y el coste de vertido de material fundido.

(iii) Reducción en el refundido.

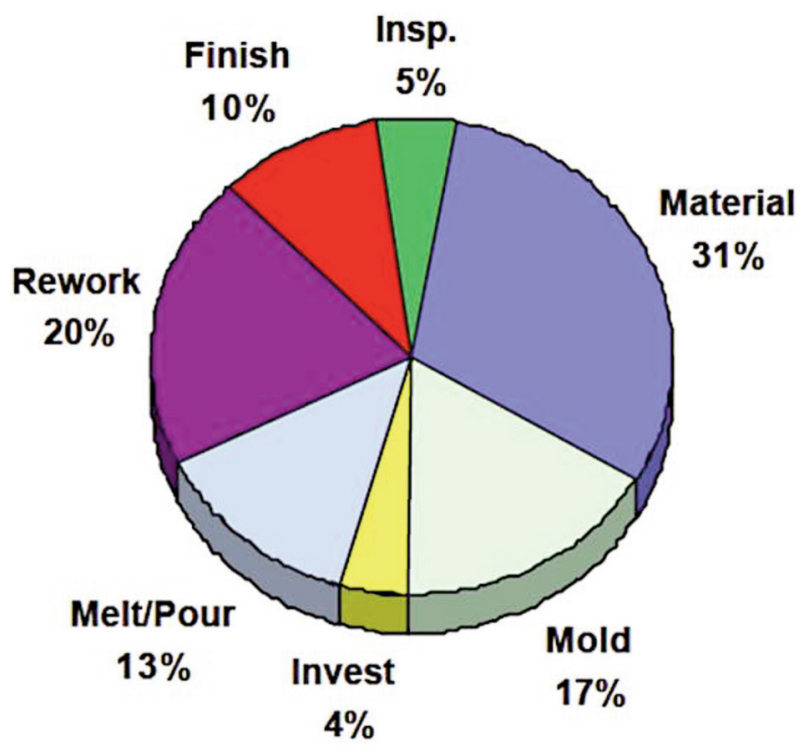

Figura 4. Análisis de la cadena de valor del proceso de fundición.

Figure 4. Value stream analysis of casting. COLD SPRAY TECHNOLOGY: INTERNATIONAL STATUS AND USA EFFORTS. Prepared by J. Karthikeyan.ASB Industries Inc. 
(iv) Reducción en el acabado.

(v) Aumento en la utilización de materiales (la proyección fría tiene una eficiencia de la deposición de $60-95 \%$ ).

Un simple cálculo, basado en los resultados de la cadena de valor ha demostrado que la entrada de materia prima puede reducirse en un $50 \%$, la reelaboración y acabado en un $75 \%$, y el molde, fundido / vertido, y los costes de fundición eliminados. Alrededor del $70 \%$ del coste de la cadena de valor podría ser reducido o eliminado. A esta reducción de costes deben añadirse los costes directos implicados en la fabricación de una pieza por proyección fría. Los costes estimados del estudio, fueron usados posteriormente en el desarrollo de casos reales para demostrar la ventaja que podía suponer el uso de la técnica de proyección fría para la fabricación de ciertas piezas.

Por otro lado, M. Grujicic et al. del centro internacional de investigación del automóvil, en colaboración con el grupo BMW realizaron un estudio económico sobre la viabilidad de la producción de una pieza híbrida polímero-metal del automóvil con las distintas técnicas de proyección. Durante la última década, las estructuras híbridas polímerometal (PMH) se han utilizado en una gran variedad de aplicaciones en el campo de la automoción, que van desde el panel de instrumentación a los parachoques.

La idea principal de este tipo de materiales es la de combinar las funciones estructurales y no estructurales de una serie de componentes, en una única pieza totalmente optimizada (por lo general consiste en un núcleo metálico recubierto por moldeo de un material plástico que contiene varias costillas.

El coste total de producción de una pieza puede dividirse en:

$$
\begin{aligned}
\mathrm{Cm}= & \text { Cmat }+ \text { Ccap }+ \text { Ctool }+ \text { Ccons }+ \\
& + \text { Cpower }+ \text { Cop }+ \text { Cmaint }
\end{aligned}
$$

Donde Cm: costes de manufactura, Cmat: costes de material, Ccap: costes de bienes de equipo, Ctool: costes de maquinaria, Ccons: coste de consumibles, Cpower: coste energético, Cop: coste mano de obra y Cmaint: coste de mantenimiento.

Basados en los resultados que se obtuvieron, se vió que el proceso de proyección fría era el método óptimo para la obtención de piezas estructurales. En la tabla I se muestra una comparación de los costes asociados a cada una de las técnicas de obtención de los recubrimientos y puede observarse cómo en el caso de la proyección fría los costes son los más bajos $^{[20]}$.

La evolución y maduración de esta tecnología establecerá la proyección fría como una técnica viable para producir recubrimientos con alto rendimiento a un precio asequible para numerosas industrias.

\section{RECUBRIMIENTOS, SUSTRATOS, RECARGUES Y CAMPOS DE APLICACION}

La proyección fría se aplica en numerosos casos, pero principalmente en aquellas situaciones en las que los recubrimientos obtenidos por las técnicas de proyección térmica (HVOF, Plasma, Llama) no son óptimos debido principalmente a las altas temperaturas que confieren al material proyectado y al sustrato. Estas altas temperaturas pueden provocar la reacción de las partículas en estado fundido, con la atmósfera o entre sí, ocasionando la aparición de óxidos o de estructuras no deseadas que inducen un cambio en las propiedades de los materiales de partida.

Tabla I. Análisis de costes comparativo

\begin{tabular}{|c|c|c|c|c|}
\hline \multirow{2}{*}{ Cost component (\$) } & \multicolumn{4}{|c|}{ Powder coating process } \\
\hline & 1. Cold-gas spray & 2. Flame spray & 3. HVOF spray & 4. Plasma-arc spray \\
\hline Material cost, $\mathrm{C}_{\text {mat }}$ & 0,3365 & 0,3883 & 0,3365 & 0,3883 \\
\hline Capital cost, $\mathrm{C}_{\text {cap }}$ & 0,0356 & 0,0148 & 0,0238 & 0,0445 \\
\hline Consumable cost, $\mathrm{C}_{\text {con }}$ & 0,2138 & 0,1438 & 1,027 & 0,0476 \\
\hline Power cost, $C_{\text {power }}$ & 0,0107 & 0,0296 & 0,0170 & 0,0391 \\
\hline Operational cost, $\mathrm{C}_{\mathrm{op}}$ & 0,2524 & 1,1649 & 0,5048 & 0,7766 \\
\hline Total cost & $0,8490 \pm 0,1274$ & $1,7415 \pm 0,2612$ & $1,9068 \pm 0,2860$ & $1,2961 \pm 0,1944$ \\
\hline
\end{tabular}

Table I. Comparative cost analysis 
Los estudios más pioneros y con una mayor inversión económica en el uso de la proyección fría, así como en la búsqueda de posibles aplicaciones, están siendo realizados principalmente por la industria militar y la aeronáutica. Pero debido a ser una técnica limpia, económicamente viable, de fácil manejo y con unas altas eficiencias permite su utilización por parte de una amplia gama de industrias como la electrónica, energética, transporte, química, consumo (materias primas, productos básicos) y nuclear entre otras.

Debido a su versatilidad, la naturaleza de los materiales que pueden ser usados para proyección fría es cada vez más amplia.

Las aplicaciones más comunes de la proyección fría son:

- Recubrimientos resistentes a la corrosión.

- Restauración y reparación.

- Recubrimientos resistentes al desgaste (degradación, erosión, fricción).

- Biomédicas (prótesis, implantes).

- Aeroespaciales.

- Recubrimientos resistentes a fatiga.

- Recubrimientos resistentes a la oxidación.

- Ampliación de la vida útil.

- Creación de superficies superconductoras, magnéticas o barreras térmicas.

El proceso de proyección fría se ha usado para producir recubrimientos densos y con buenos valores de adherencia de numerosos metales (alumunio, cobre, níquel, titanio, plata y zinc), aleaciones (aceros inoxidables, inconels, MCrAlYs, hastelloys), composites (cermets, $\mathrm{Al}-\mathrm{Al}_{2} \mathrm{O}_{3}, \mathrm{Al}$-SiC, WC-Co) e intermetálicos entre otros. En la figura 5 pueden observarse algunos recubrimientos obtenidos por proyección fría.

El aluminio, junto con el cobre, el titanio y el zinc, son los metales sobre los que un mayor número de estudios se han realizado. Esto es debido a la combinación de sus propiedades junto con la buena calidad de las deposiciones obtenidas en su proyección.

La proyección fría ha resultado ser un método muy efectivo para adherir partículas de aluminio sin la necesidad de fundirlas ${ }^{[21]}$. El mecanismo principal de adhesión observado sugiere una unión mecánica de las partículas con el sustrato y entre sí.

Los recubrimientos de aluminio y de sus aleaciones son principalmente investigados para su uso como elemento de reparación o recargue en la industria aeroespacial, como material frente a la corrosión en la industria petroquímica así como elemento de aporte en procesos de soldadura ${ }^{[21]}$, J.M. Guilemany et al. han obtenido recubrimientos densos, de gran espesor y con buenos valores de adherencia de aluminio sobre sustratos de acero al carbono ${ }^{[22]}$.

Se han realizado investigaciones del uso del aluminio para producir recubrimientos conductores sobre sustratos no metálicos como los cerámicos. Debido a su frágil naturaleza, el uso de este tipo de sustratos implica un reto para la técnica de proyección. La metalización de materiales aislantes para aplicaciones electrónicas haciendo uso de la proyección fría ha resultado ser una aplicación viable. Peter C. King et al. han llegado a producir recubrimientos de aluminio sobre sustratos piezocerámicos (PZT). Un material piezocerámico requiere una conversión eficiente de energía mecánica en eléctrica o viceversa.
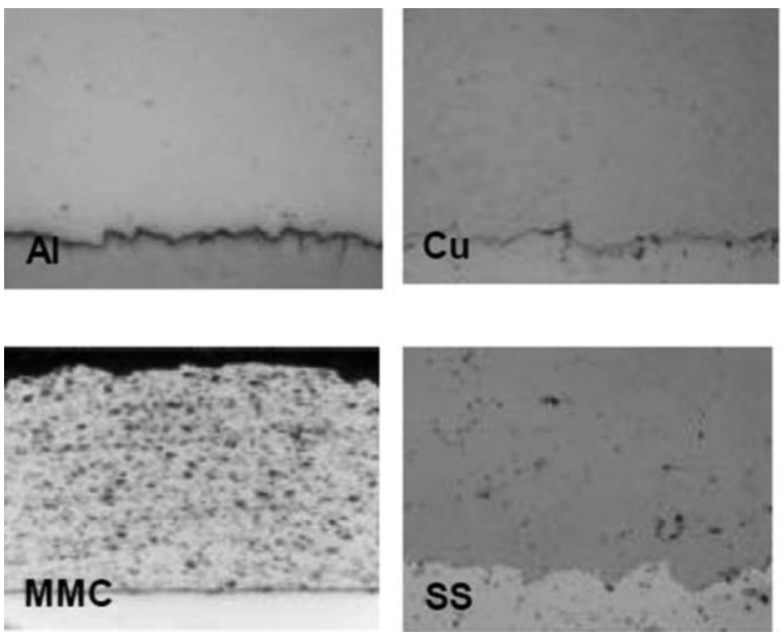
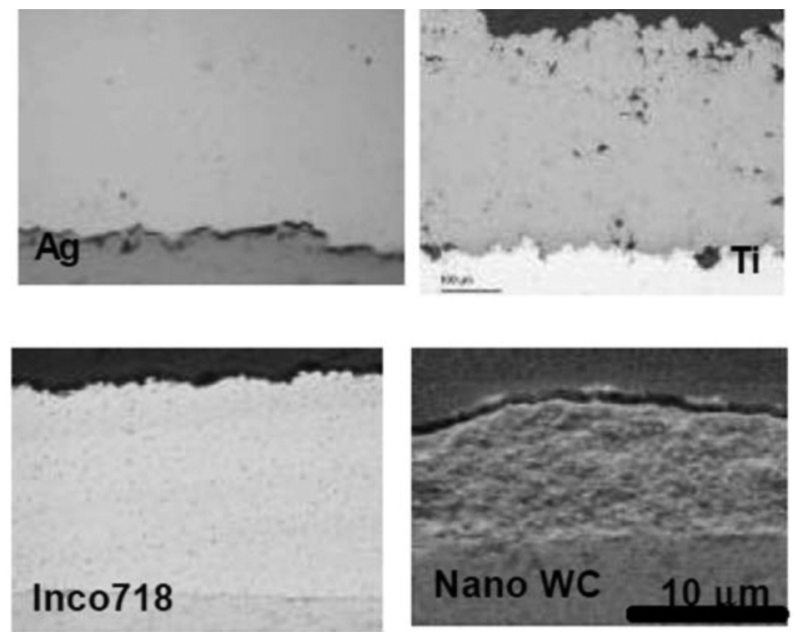

Figura 5. Recubrimientos obtenidos por proyección fría.

Figure 5. Cold gas spray coatings. 
Electrodos metálicos en caras opuestas al elemento piezocerámico permiten la aplicación de un campo eléctrico uniforme. La proyección fría no afecta a las propiedades del sustrato incluso en elementos polarizados. El espesor de los recubrimientos obtenidos es mayor que los conseguidos por la técnica de esputerizado y con una resistividad eléctrica del aluminio proyectado de $9,9 \pm 0,5 \mu \Omega \mathrm{cm}^{-1}[23]$.

Durante el último medio siglo, el titanio ha ido adquiriendo una mayor importancia en el sector industrial. Esto se debe principalmente a su alta resistencia específica, su alta resistencia a la fatiga y a la fractura, y su buena resistencia a la corrosión. Estas propiedades, combinadas con su baja densidad (aproximadamente un $55 \%$ de la densidad del acero) hacen que las aleaciones de titanio sean muy utilizadas para fabricar componentes aeroespaciales que trabajen bajo altas cargas y en un amplio intervalo de temperaturas (desde bajas a moderadas), esto incluye desde fuselajes a componentes de motores a reacción.
La resistencia a la corrosión del titanio y sus aleaciones es debida a la capa estable de óxido que se forma en la superficie y que aísla el material del medio externo. Esta propiedad permite utilizar el titanio en industrias con medios más agresivos como la industria química o la biomédica.

La proyección fría como técnica de deposición para la obtención de recubrimientos de titanio ha ido en aumento ya que no se necesitan atmósferas protectoras o vacio para realizar los recubrimientos ${ }^{[24]}$. S. Gulizia et al. han obtenido recubrimientos densos de titanio puro sobre un sustrato de aluminio Al-6061 con una porosidad del $1 \%$, una dureza ligeramente superior a la del polvo de partida y donde todas las tensiones existentes en el recubrimiento son de tipo compresivo.

KeeHyun Kim et al. depositaron titanio sobre tres tipos de sustratos de distinta naturaleza viendo cómo en función de las propiedades del sustrato, el enlace y la deformación de las partículas proyectadas eran diferentes ${ }^{[25]}$.

Los resultados, expuestos en la figura 6, demostraron que el comportamiento de deformación de las
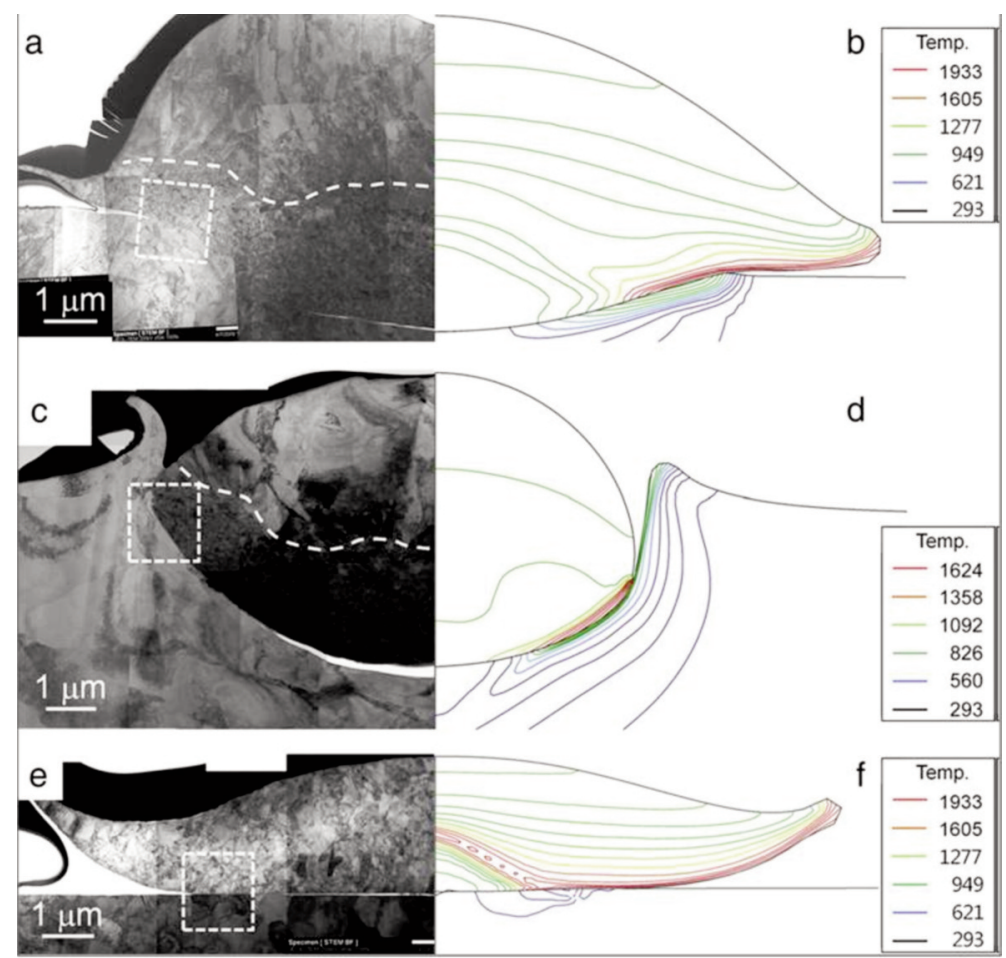

Figura 6. Imágenes transversales STEM y distribuciones de la temperatura de cada partícula de titanio depositado en titanio $(a, b)$, aluminio $(c, d)$ y óxido de zirconio $(e, f)$.

Figure 6. STEM cross-sectional images and temperature distributions of each particle of titanium deposited on titanium $(a, b)$ aluminium $(c, d)$ and zirconium oxide $(e, f)$. Bonding mechanisms of thermally softened metallic powder particles and substrates impacted at high velocity. KeeHyun Kim et al. 
partículas y los sustratos eran influenciados principalmente por la dureza del sustrato, mientras que las microestructuras internas reflejaban tanto la deformación como la temperatura local de ambos materiales.

Otros autores, emplean helio como gas transportador para disminuir la porosidad de los recubrimientos debido a que la capacidad de transmisión térmica del gas es mayor. Otros parámetros que influyen en la disminución de la porosidad son una menor distancia de proyección así como una distribución de tamaño de partícula menor. La variación de estos parámetros ha dado lugar a recubrimientos de titanio densos y con una dureza mayor a la del material base ${ }^{[26}$ y 27$]$.

Otro modo de obtener recubrimientos con un menor porcentaje de porosidad, es realizar tratamientos térmicos tras la deposición. Según T. Hussain et al. esta disminución en la porosidad debida al tratamiento térmico es provocada por el crecimiento de los granos ocasionado por las altas temperaturas ${ }^{[28]}$. Se han llegado a obtener recubrimientos de titanio densos de hasta $3 \mathrm{~mm}$ de espesor sobre aleaciones de aluminio Al-6063, con buena adherencia y con una dureza superior a la del material base debido al trabajo en frío producido durante la deposición.

J.M. Guilemany et al. realizaron estudios comparativos variando las condiciones de presión, temperatura y tasa de alimentación de polvo, para ver su influencia en la calidad de los recubrimientos de titanio sobre sustratos de aluminio 7075 T6. Obtuvieron recubrimientos densos y con una porosidad menor al
$1 \%$ en las condiciones óptimas de proyección. Los recubrimientos de la figura 7 , tienen unas durezas alrededor de los $250 \mathrm{HV}$ llegando a duplicar los valores de dureza del sustrato ${ }^{[29]}$, además de aumentar la resistencia al desgaste por fricción y abrasivo ${ }^{[30]}$.

Del mismo modo que en el caso del aluminio, D. Rafaja et al. han obtenido recubrimientos densos de titanio con buena adherencia sobre sustratos de alúmina. Durante el impacto sobre el sustrato, el titanio se deforma plásticamente y rellena las cavidades en la superficie del sustrato. Este anclaje mecánico entre el recubrimiento de titanio y el sustrato de alúmina es considerado como uno de los motivos de los buenos resultados de adherencia, entre otros ${ }^{[31]}$. Las ventajas que la técnica de proyección fría aporta, permiten realizar recubrimientos de titanio nanoestructurado bien adheridos y homogéneos. Las deformaciones producidas en las partículas así como los perfiles de temperatura, pueden verse en la figura 8 que es una simulación obtenida por G. Baea et al. ${ }^{[32]}$.

Tal como se presenta en este trabajo, una de las principales ventajas que ofrece la tecnología de CGS es la ausencia de oxidación de la materia prima. Este aspecto es crítico en el caso del titanio y hace del CGS una técnica prometedora y competitiva en las prótesis de ingeniería médica. El titanio es conocido por sus propiedades biocompatibles, es decir, permanece inalterable cuando se implanta en el cuerpo humano. El objetivo principal de los estudios realizados por J.M. Guilemany et al. era la producción de recubrimientos porosos de titanio grado 2 no oxidado, con buenos valores de adherencia y capacidad de

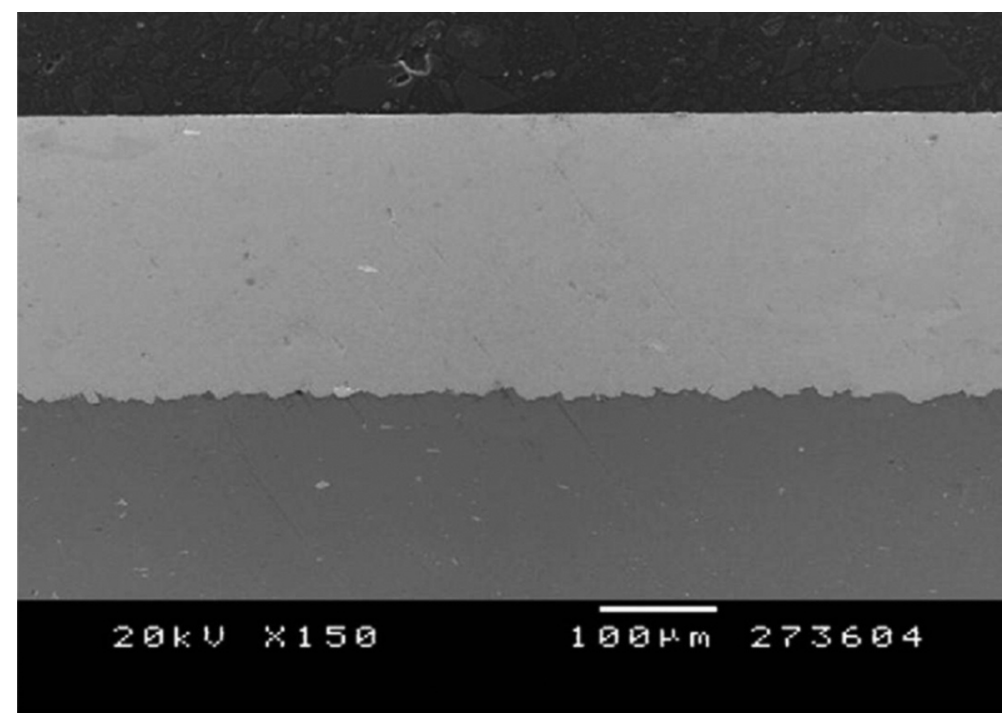

Figura 7. Recubrimiento de Ti grado 2 sobre Al7075-T6 en las condiciones óptimas de CGS.

Figure 7. Ti grade 2 coating on Al7075-T6 in optimum CGS conditions. 

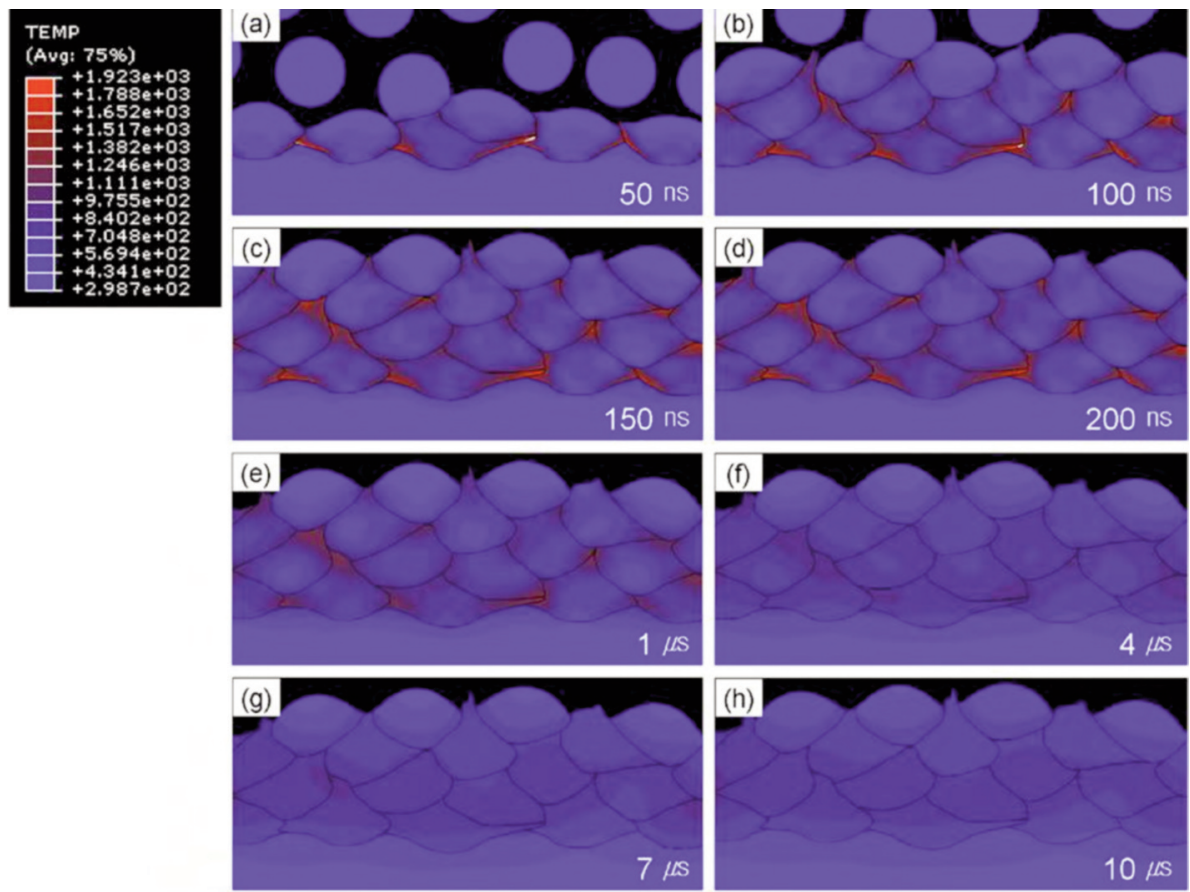

Figura 8. Simulaciones de la deposición de partículas de titanio sobre un sustrato de acero a distintos tiempos ( los patrones de deformación y la temperatura (grados Kelvin) en los perfiles (a) 50 ns, (b) $100 \mathrm{~ns}$, (c) $150 \mathrm{~ns}$, (d) $200 \mathrm{~ns}$, (e) $1 \mu \mathrm{s}$, (f) $4 \mu \mathrm{s}$, (g) $7 \mu \mathrm{s}$ y $10 \mu \mathrm{s}$ (h)).

Figure 8. Simulations of particle deposition of titanium on a steel substrate at different times (the deformation patterns and temperature (Kelvin) in the profiles (a) $50 \mathrm{~ns}$, (b) $100 \mathrm{~ns}$, (c) $150 \mathrm{~ns}$, (d) $200 \mathrm{~ns}$, (e) $1 \mu \mathrm{s}$, (f) $4 \mu \mathrm{s}$, (g) $7 \mu \mathrm{s}$ y $10 \mu \mathrm{s}(\mathrm{h}))$. Nanostructure formation and its effects on the mechanical properties of kinetic sprayed titanium coating G. Bae.

integrarse completamente con el hueso. En la figura 9 puede observarse una prótesis vertebral recubierta de titanio grado 2 por la técnica de CGS.

Tal y como se ha comentado anteriormente, la técnica permite la proyección de materiales de muy diversa naturaleza debido a sus propiedades o a las aplicaciones que se pueden derivar de dichas propiedades.

En el caso del cobre, su principal aplicación es la obtención de recubrimientos conductores o como protección frente a la corrosión. HJ. Choi et al. han desarrollado un nuevo método para la fabricación de contenedores para la eliminación de combustible. Los contenedores de hierro fundido están recubiertos de una capa de cobre para evitar la corrosión. El cobre ha sido depositado por proyección fría permitiendo un grosor de la capa de $10 \mathrm{~mm}$. Este método permite el ahorro de más de 6 toneladas de cobre al pasar de un espesor de capa de $50 \mathrm{~mm}$ a otro de $10 \mathrm{~mm}{ }^{\text {[33] }}$. En la figura 10, puede verse un esquema de la obtención de dicho recubrimiento.
$\mathrm{Al}$ igual que en el caso del aluminio y del titanio, se ha proyectado cobre sobre sustratos cerámicos obteniendo resultados muy prometedores. En la industria del automóvil, los módulos electrónicos de potencia se usan en convertidores, unidades de control, etc.. Un sustrato electrónico estándar para estos módulos, consiste en una placa cerámica recubierta de una capa de doble cara de cobre como se observa en la figura 11. B. Wielage et al. han obtenido recubrimientos densos, exentos de óxidos y bien adheridos de $\mathrm{Al} / \mathrm{Cu}$ sobre sustratos de $\mathrm{Al}_{2} \mathrm{O}_{3}$. El cobre es el material conductor, mientras que el aluminio actúa como una capa de anclaje entre el sustrato cerámico y el cobre ${ }^{[34]}$.

Otra aplicación interesante del cobre es su deposición como capa conductora sobre plásticos. Debido a la baja temperatura de las partículas de proyección, se pueden obtener recubrimientos metálicos con buenos espesores en materiales termoplásticos como la poliamida ${ }^{[35]}$.

Los recubrimientos de níquel son ampliamente usados para producir capas protectoras frente a la 


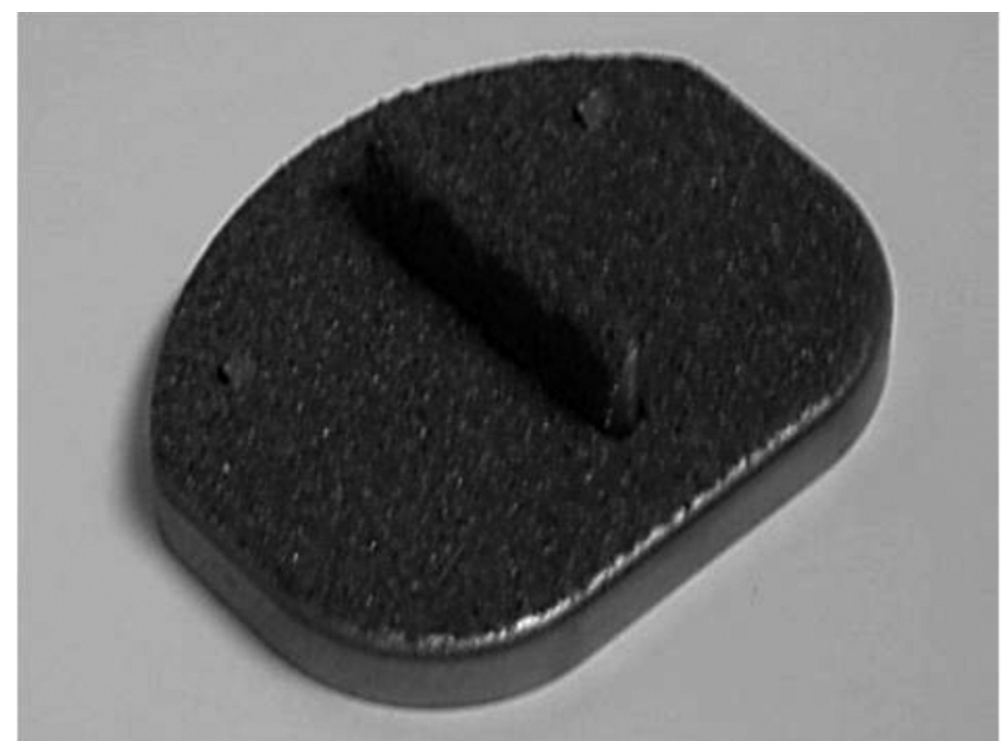

Figura 9. Prótesis de rodilla recubierta de titanio grado 2 por CGS.

\section{Figure 9. Knee Prostheses coated with titanium grade 2 by CGS.}

corrosión y al desgaste. Otra aplicación del níquel o de los metales ferromagnéticos y sus aleaciones depositados por la técnica de proyección fría, es la forma-

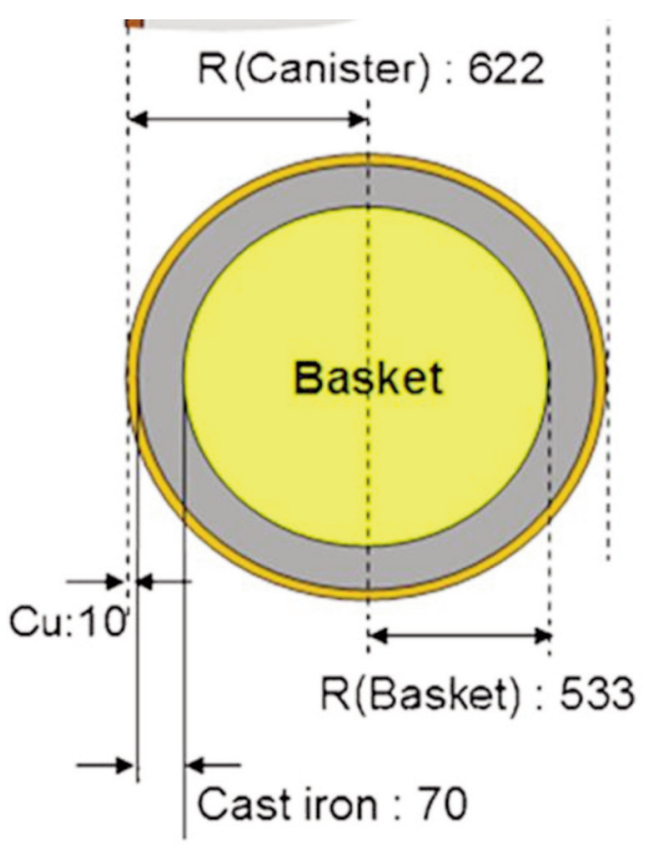

Figura 10. Esquema del recubrimiento de cobre obtenido por CGS.

Figure 10. Copper coating obtained by CGS. Application of a cold spray technique to the fabrication of a copper canister for the geological disposal of CANDU spent fuels Heui-Joo Choi et. al. ción de recubrimientos para utensilios de cocina (calentamiento por inducción).

Otro metal que está cobrando importancia es el zinc. S. Cadney et al. han planteado el uso de zinc como material de aporte para fabricar formas volumétricas así como en soldadura. En ambos casos el sustrato empleado fue la aleación de aluminio 6061 como puede observarse en la figura 12. Los valores de dureza obtenidos para ambas aplicaciones fueron similares ${ }^{[36]}$.

K. Onizawa et al. han obtenido recubrimientos de Zn, Zn-Al y Zn-Mg para aplicaciones de impresión, densos y con durezas de hasta cuatro veces superiores a las de una pieza de zinc. Estos nuevos desarrollos abren nuevas oportunidades de producir recubrimientos más duros y más resistentes que permiten la producción de un mayor número de copias sin comprometer a la calidad ${ }^{[37]}$.

Uno de los metales más innovadores que se está proyectando por esta técnica es el tántalo. Es un metal refractario con un alto punto de fusión $\left(\mathrm{T}_{\mathrm{f}}=2.996^{\circ} \mathrm{C}\right)$ fácilmente mecanizable y una baja temperatura de transición dúctil-frágil. A causa de su alta resistencia a la corrosión a medios ácidos (excepto HF) y salinos, así como a productos químicos orgánicos debido a la capa de pasivación que se forma en la superficie $\left(\mathrm{Ta}_{2} \mathrm{O}_{5}\right)$, es un material muy apreciado. $\mathrm{H}$. Koivuluoto et al. han obtenido recubrimientos densos e impermeables de tántalo sobre sustratos de acero, aluminio y cobre, que actúan como barreras frente a la corrosión $^{[38]}$. 


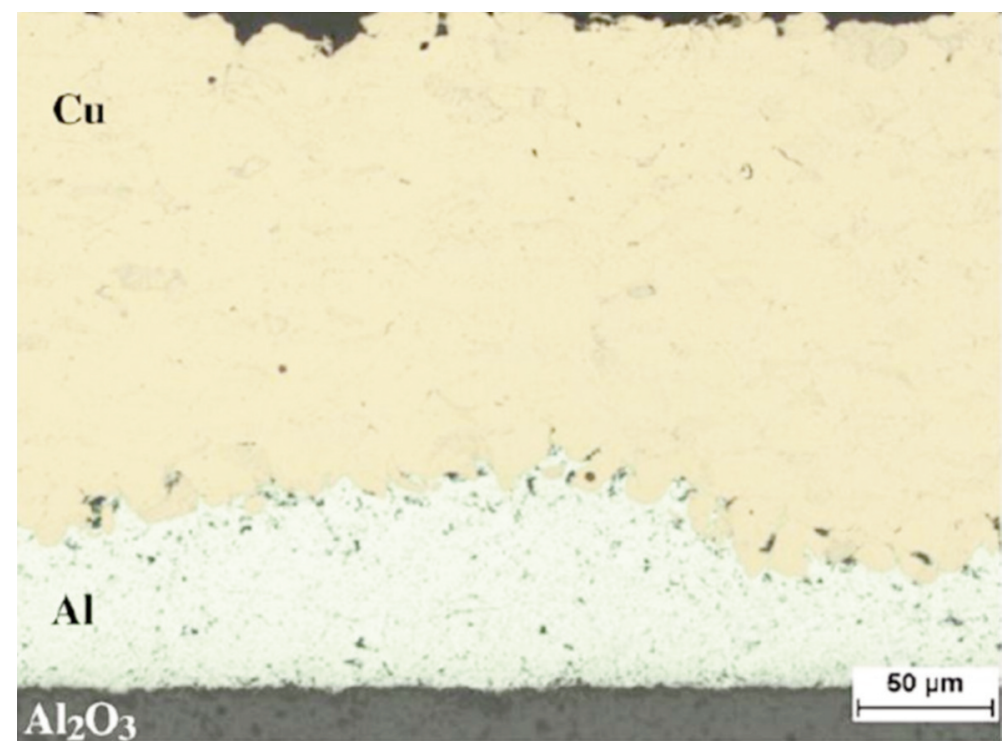

Figura 11. Recubrimiento conductor de cobre obtenido por CGS.

Figure 11. Copper conductive coating obtained by CGS. New method for producing power electronic circuit boards by coldgas spraying and investigation of adhesion mechanisms Wielage et. al.
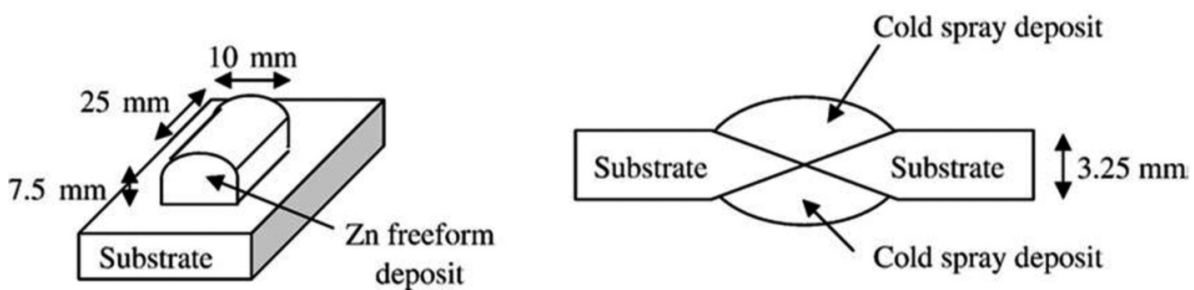

Figura 12. Recargues de Zn por CGS.

Figure 12. Zn Recharge by CGS. Cold gas dynamic spraying as a method for freeforming and joining materials. S. Cadney et. al.

R.Lupoi et al. han realizado recubrimientos de cobre, aluminio y estaño sobe una amplia gama de sustratos como PC / ABS, poliamida-6, polipropileno, poliestireno y de un material compuesto de fibra de vidrio. La naturaleza del sustrato hace que el proceso de deposición sea particularmente difícil. En la figura 13, puede verse que la ventana de deposición es mucho menor que en el caso de los sustratos metálicos o cerámicos ${ }^{[39]}$.

La técnica de proyección fría tiene una gran versatilidad: permite no sólo proyectar un único tipo de polvo, sino dos al mismo tiempo. Sova et al. han realizado proyecciones de $\mathrm{Cu}+\mathrm{Al}$. Para ello utilizaron una pistola de proyección con doble entrada para polvo, lo que permite introducir primero uno de los materiales, en este caso el cobre, y a conti- nuación, el segundo material que es el aluminio. La composición del volumen de la mezcla de proyección fue de aproximadamente $60 \%$ de aluminio y $40 \%$ de cobre. Se obtuvo una densa capa de cobre y aluminio ${ }^{[40]}$.

Otro método de proyección de dos polvos al mismo tiempo, en vez de con una pistola de proyección de doble entrada, es mezclar los polvos en la tolva. Xian-Jin Ning et al. han realizado proyecciones de aluminio con un 10 y un $20 \%$ de estaño sobre un sustrato de Al6061 y sobre otro de SUS304. Obtuvieron recubrimientos densos y homogéneos de ambas mezclas usando helio como gas transportador. Esta mezcla de aluminio y estaño es muy utilizada en la industria automovilística como material antifricción ${ }^{[41]}$. 


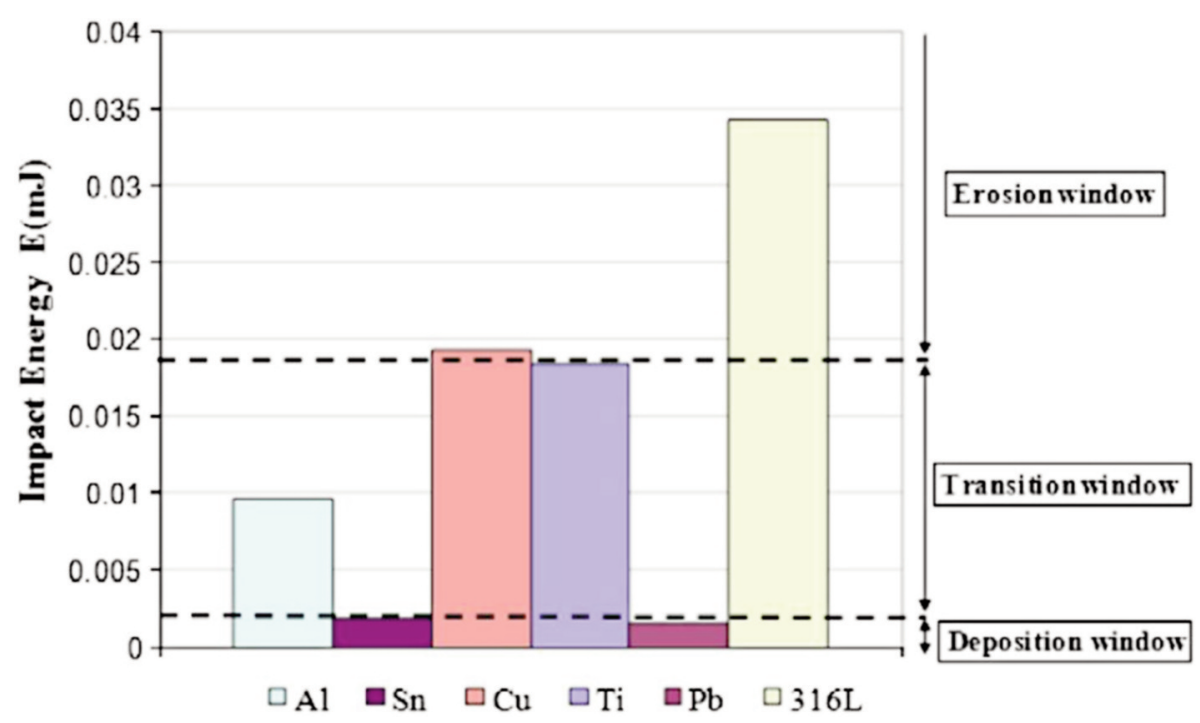

Figura 13. Proyección fría de distintos materiales metálicos sobre materiales poliméricos.

Figure 13. Cold spray process initial characterization chart for polymer substrates. Deposition of metallic coatings on polymer surfaces using cold spray R. Lupoi.

Una de las aleaciones más utilizadas son los aceros inoxidables, entre ellos el 316. C.Lorenzana et al. han obtenido recubrimientos de este tipo de acero con muy buenos resultados de adherencia (por encima de $50 \mathrm{MPa}$ ) y de dureza, después de haber optimizado los parámetros más influyentes en el proceso de proyección ${ }^{[42]}$.

Las aleaciones metálicas más conocidas son aquéllas que actúan como barreras térmicas que son usadas para proteger y aislar componentes metálicos en las zonas de alta temperatura de turbinas de gas en aviones y plantas energéticas. El uso de estos materiales da lugar a una reducción de temperatura de entre 100 y $300^{\circ} \mathrm{C}$ sobre la superficie del sustrato metálico y por lo tanto, aumentan la duración de los componentes metálicos y mejoran el rendimiento del motor ${ }^{[43-47]}$.

N.Cinca et al. del Centro de Proyección Térmica obtuvieron recubrimientos de stellite denso y sin presencia de óxidos, representados en la figura 14, mejorando las propiedades de dureza, resistencia a la abrasión, fricción y resistencia a la oxidación con respecto al sustrato de acero inoxidable usado ${ }^{[48]}$.

Otras aleaciones empleadas en proyección fría son los vidrios amorfos metálicos base hierro. Estos tipos de materiales presentan altas resistencias, bajos coeficientes de fricción, altas resistencias a la corrosión y al desgaste, buenas propiedades magnéticas y un bajo coste de material para aplicaciones indus- triales. S. Yoona et al. han obtenido buenos recubrimientos usando helio como gas transportador y precalentando el material a depositar ${ }^{[49]}$.

El crecimiento en la demanda de producción de piezas y estructuras con menor peso, resistentes y que puedan trabajar a elevadas temperaturas, ha provocado un gran aumento en el uso de aleaciones ligeras principalmente aquellas procedentes del aluminio o el magnesio.

A pesar de las grandes ventajas que estas aleaciones representan para la industria, su gran inconveniente son las limitaciones en las propiedades mecánicas para su uso en numerosas industrias clave, como puede ser la aeronáutica o la industria petroquímica. Esta misma necesidad es la que apoya el uso de la proyección fría debido principalmente a la característica de mantener la estructura de los polvos de partida así como las características del sustrato ${ }^{[50]}$. De este modo, se pueden modificar las propiedades superficiales de las piezas sin perder las propiedades de las aleaciones ligeras.

Los materiales intermetálicos juegan un papel muy importante en la protección superficial de este tipo de aleaciones. Diversos autores han obtenido recubrimientos de materiales intermetálicos por proyección fría.

Existen dos líneas principales de obtención de los recubrimientos intermetálicos de $\mathrm{Ni}$ - $\mathrm{Al}$ cuyas principales características son la resistencia a la temperatura y a la corrosión ${ }^{[51]}$. 


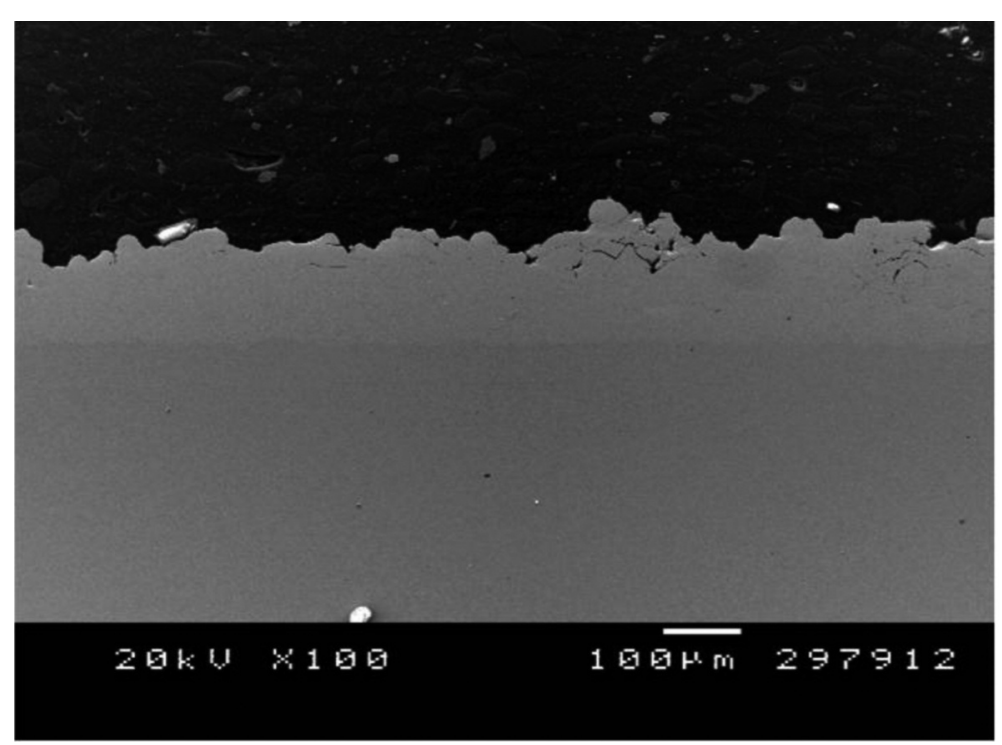

Figura 14. Recubrimiento de stellite obtenido por CGS.

Figure 14. Stellite coating obtained by CGS.

- Proyección de ambos polvos sobre un sustrato y tratamiento térmico posterior ${ }^{[52]}$.

- Proyección de aluminio sobre un sustrato de níquel o viceversa y un posterior tratamiento térmico para la obtención del intermetáli$\cos ^{[53]}$.

Del mismo modo que se han obtenido los intermetálicos de Ni-Al, S. Tria et al. han obtenido recubrimientos intermetálicos de $\mathrm{Ni}$ - Ti por proyección directa del material y sin tratamiento térmico posterior para aplicaciones médicas y estructuras inteligentes debido a propiedades de superelasticidad, memoria de forma y buena amortiguación ${ }^{[54]}$, mientras que $\mathrm{T}$. Ando et al. han obtenido recubrimientos de $\mathrm{Nb}_{3} \mathrm{Al}$ por su interés como superconductor para bobinas $^{[55]}$.

La obtención de recubrimientos por proyección fría de materiales compuestos (composites) y dentro de éstos, los "cermets" (cerámico+metal) no es sencilla, pero es un método recomendable ya que evita la degradación y la descarburización de las fases no metálicas en comparación con otros métodos de proyección térmica como la alta velocidad (HVOF) o la proyección por plasma (APS). En este tipo de compuestos, la componente metálica actúa como una matriz que retiene a las partículas de refuerzo que le confieren unas propiedades muy determinadas.

W.Y.Li et al. obtuvieron recubrimientos de Al2319 reforzados con partículas de TiN llegando a conseguir un aumento del $45 \%$ en dureza, así como un aumento de más del $60 \%$ en adherencia con respecto a un recubrimiento de la aleación de aluminio pura, así como un incremento significativo de resistencia al desgaste ${ }^{[56]}$.

El $\mathrm{Al}_{2} \mathrm{O}_{3}$ es uno de los compuestos cerámicos más usados para el refuerzo de matrices metálicas, las cuales pueden ser de aluminio, níquel etc. ${ }^{[57-59]}$.

J.M.Guilemany et al. han conseguido recubrimientos compuestos de bronce/alúmina con valores de dureza y resistencia al desgaste superiores a aquellos recubrimientos obtenidos por APS. En la figura 15 puede observarse un ejemplo de uno de los recubrimientos de bronce/alúmina obtenido por la técnica de CGS [60].

Otros compuestos comúnmente usados como refuerzo son los carburos. La principal aplicación de los recubrimientos de carburos metálicos es su uso como protección al desgaste. La dureza de las partículas de WC son las que mayoritariamente confieren esta resistencia al desgaste, mientras que la matriz de cobre le confiere tenacidad ${ }^{[61-64]}$.

El proceso de proyección fría es un método alternativo a la proyección térmica convencional para proyectar no sólo carburos metálicos o cermets de tamaño micrométrico sino también materiales nanoestructurados, debido a su baja temperatura durante el proceso, conservando la nanoestructura del polvo original durante el proceso.

J.S. Kim et al. realizaron numerosos estudios para conocer la microestructura de recubrimientos obtenidos por proyección fría de polvo de $\mathrm{TiB}_{2}-43 \%$ vol. Cu que contenía partículas de diboruro de titanio con un tamaño de $50-100 \mathrm{~nm}$. Este tipo de material es un buen candidato para 


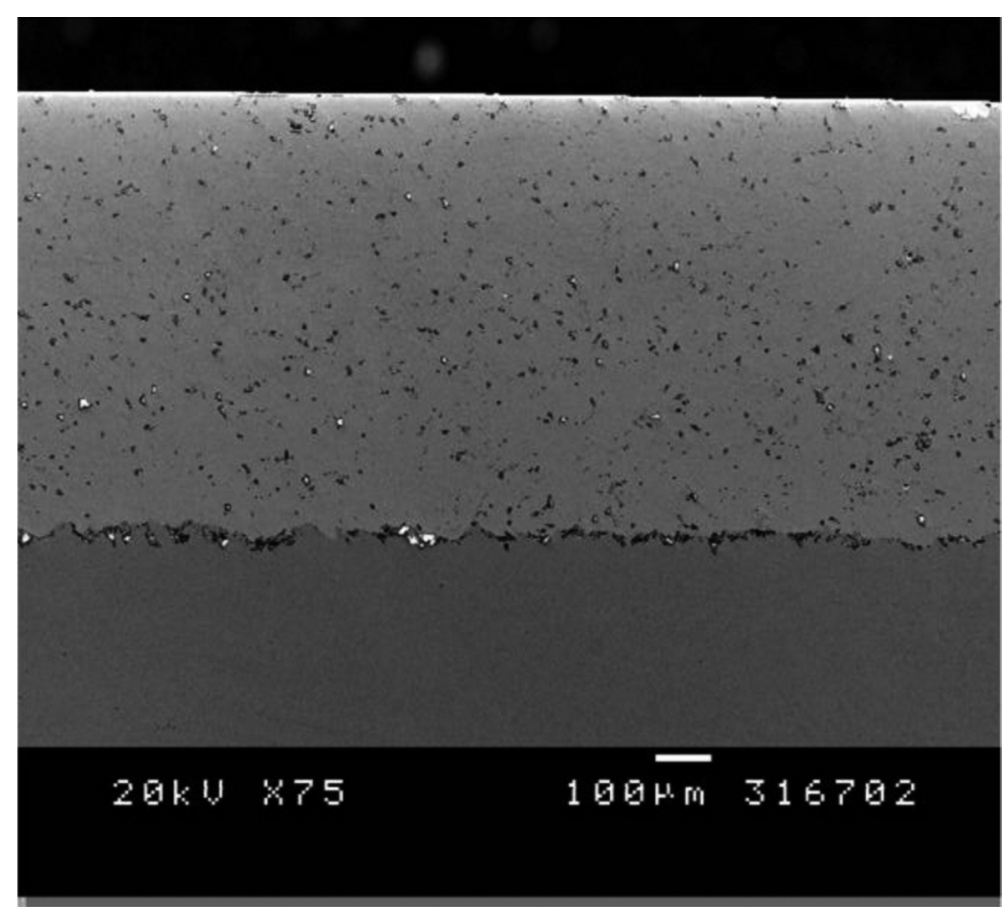

Figura 15. Imagen de la pieza mecanizada PF-3 \%.

Figure 15. Image of the sample PF- $3 \%$ after machining.

aquellas aplicaciones que requieran materiales que combinen una buena resistencia mecánica, resistencia al desgaste y conductividad térmica y eléctrica. Por lo tanto, el sistema $\mathrm{TiB}_{2}$-Cu es muy útil para aplicaciones tales como contactos deslizantes, puntas de electrodos para soldadura por resistencia, interruptores, etc. ${ }^{[65]}$.

Hasta el momento se ha visto que todos los materiales proyectados son metálicos o una proporción muy alta del polvo lo es, pero también se han realizado algunas pruebas utilizando polvos de naturaleza distinta.

M.Yamada et al. así como J.O. Klieman et al. han usado la técnica de proyección fría para depositar dióxido de titanio, que es un cerámico para su aplicación como capa fotocatalítica. El dióxido de titanio es un prometedor fotocatalizador debido a sus posibles aplicaciones en la purificación del medioambiente, células solares, esterilización, terapias anticancerígenas y capas antiniebla ${ }^{[66}$ y 67].

El óxido de titanio no es el único material cerámico con el que se ha conseguido producir un recubrimiento por proyección fría. Feng Wang et al. realizaron recubrimientos de $\mathrm{NiO} / \mathrm{Al}_{2} \mathrm{O}_{3}$ para su uso como recubrimiento catalizador en la producción de hidrógeno ${ }^{[68]}$.

La proyección fría es una técnica que no solo se usa para generar recubrimientos sino también como sistema de recargue o como método de soldadura.
J. C. Lee et al. dañaron a propósito la superficie de un molde de forma mecánica para representar los posibles daños potenciales que pudiese sufrir el molde durante el mecanizado. Más tarde, se depositó por proyección fría aluminio puro sobre la parte dañada. Por último, se mecanizó de nuevo el molde para recuperar la forma original (Fig. 16) ${ }^{[69]}$.

Los componentes de las turbinas a alta presión incluidos los álabes, son componentes críticos en cualquier motor de turbina. Durante el funcionamiento del motor de una turbina, los componentes de alta presión están sometidos a altas temperaturas y a cargas de tensión. Esta alta temperatura junto con la tensión, puede dar lugar a tasas de degradación de los componentes de la turbina inaceptablemente altos, causados por erosión, oxidación, corrosión, fracturas por fatiga térmica y daños por objetos extraños. Estas condiciones de trabajo dan lugar a una necesidad de reparación y/o reemplazo, lo que produce importantes gastos de explotación y un tiempo fuera de servicio.

V.F. Kosarev et al. realizaron reparaciones en las láminas de turbina, paletas y otros componentes usando la proyección fría. A continuación se realizó un post-tratamiento para consolidar y homogeneizar el material depositado y así restaurar íntegramente las propiedades del material en el componente de la turbina reparado ${ }^{[70]}$. 


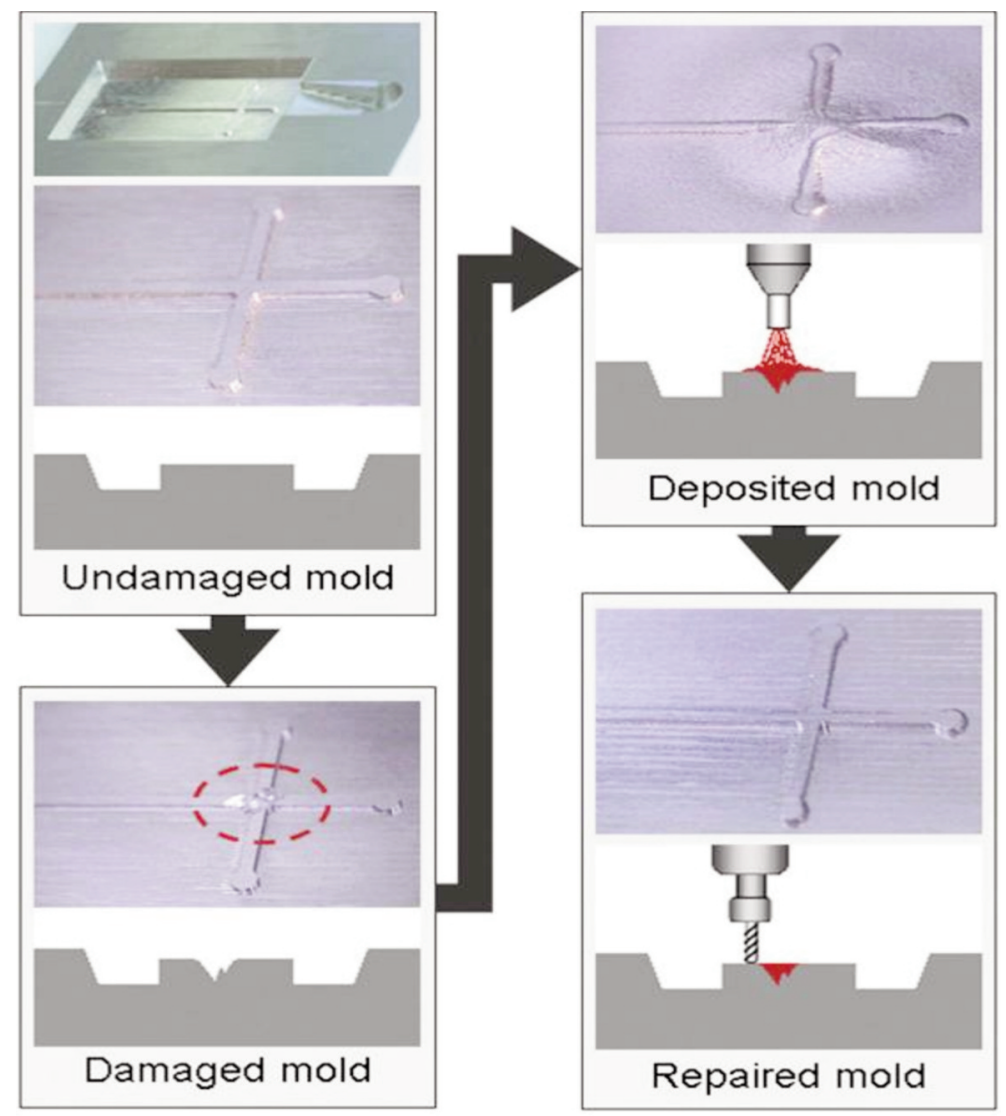

Figura 16. Reparación del molde por CGS.

Figure 16. Mold repair by CGS. Repair of Damaged Mold Surface by Cold-Spray Method J. C. Lee et. al.

\section{CONCLUSIONES}

- Como ha podido verse a lo largo del trabajo, la proyección fría ha ampliado mucho el campo de los sectores en los que la proyección térmica tenía acceso pero en condiciones limitadas.

- Sus aplicaciones son cada vez mayores, así como el número de sectores y empresas que lo utilizan.

- La técnica de proyección fría se ha establecido como una técnica viable y económicamente efectiva frente a otras tecnologías en el ámbito de la ingeniería de superficies.

- De las investigaciones llevadas a cabo, se puede concluir que la proyección fría se está utilizando para depositar un amplio número de materiales (metales, polímeros, composites e incluso cerámicos) sobre todo tipo de sustratos.

- La proyección fría tiende a verse como una gran solución a numerosos problemas que hasta ahora generaban grandes costes, mucho tiempo y fuerte agresión medioambiental con técnicas convencionales.

\section{Agradecimientos}

Los autores agradecen al Ministerio de Ciencia e Innovación la concesión del proyecto MAT 2009-10827 así como a la Generalitat de Catalunya el soporte económico recibido en el proyecto 2009SGR00390. La autora María Villa Vidaller agradece al Centro de Proyección Térmica la beca recibida.

\section{REFERENCIAS}

[1] F. Gärtner, T. Stoltenhoff, T. Schmidt y H. Kreye, J. Therm. Spray Techn. 15 (2003) 223-232.

[2] R. Ghelichi, M. Guagliano R. Ghelichi y Alli, Frattura ed Integrità Strutturale 8 (2009) 30-44.

[3] H. Assadi, F. Gärtner, T. Stoltenhoff y H. Kreye, Acta Mater. 51 (2003) 4.379-4.394.

[4] G. Bae, S. Kumar, S. Yoon, K. Kang, H. Na, H-J. Kim y C.Lee, Acta Mater. 57 (2009) 5654-5666.

[5] A.N. Papyrin, European Summer University, ENISE, St-Etienne, France, September 2006, pp 11-15. 
[6] M. Grujicic, C.L. Zhao, W.S. Derosset y D. Helfritch, Mater. Design. 25 (2004) 681-688.

[7] W-Y. Li, C. Zhang, X. Guo, C-J. Li, H. Liao y C. Coddet, Appl. Surf. Sci. 254 (2007) 517-526.

[8] S. Vladimirovich, V. Fedorovich y M. Rein, Aerosp. Sci. Technol. 9 (2005) 582-591.

[9] T. Schmidt, F. Gärtner, H. Assadi y H. Kreye, Acta Mater. 54 (2006) 729-742.

[10] M.M. Hyland, Y.-C. Lau, C.-J. Li, R.S. Lima y G. Montavon, (Ed.) B.R. Marple ISBN: 978 0-387-77285-1.

[11] B. Jodoin, L. Ajdelsztajn, E. Sansoucy, A. Zuñiga, P. Richer y E.J. Lavernia, Surf. Coat. Tech. 201 (2006) 3.422-3.429.

[12] E. Irissou, J.G. Legoux, C. Moreau, Boucherville y A. N. Ryabinin, International Thermal Spray Conference \& Exposition 2008: Thermal Spray Crossing Borders (DVS-ASM) 2008, pp. 613-619.

[13] M. Grujicic, C.L. Zhaoa, C. Tonga, W.S. Derosset y D. Helfritch, Mater. Sci. Eng. A368 (2004) 222 230.

[14] A.N. Papyrin, S.V. Klinkov y V.F. Kosarev, ITSC 2005: Thermal Spray connects: Explore its surfacing potential (DVS-ASM) pp. 145-150.

[15] Li Gang, Wang Xiao-Fang y Li Wen-Ya, Trans. Nonferrous Met. SOC. China 17(2007) 116-121.

[16] J. Pattison, S. Celotto, A. Khan y W. O'Neill, Surf. Coat. Tech. 202 (2008) 1.443-1.454.

[17] P.C. King y M. Jahedi, Appl. Surf. Sci. 256 (2010) 1.735-1.738.

[18] K. Taylor, B. Jodoin, J Karov y P. Richer, J. Therm. Spray Tech., Volume 15(2) June (2006) 273-279.

[19] Prepared By: J. Karthikeyan, ABS Industries Inc., December 2004.

[20] M. Grujicic, V. Sellappana, L. Mearsa, X. Xuana, N. Seyrb, M.Erdmann y J.Holzleitnerc, J. Mater. Process. Tech. 198 (2008) 300-312.

[21] R. Morgan*, P. Fox, J. Pattison, C. Sutcliffe y W. O’Neill, Materials Letters 58 (2004) 1.317-1.320.

[22] S. Dosta, C. Lorenzana, N. Cinca y J.M. Guilemany. $18^{\text {as }}$ Jornadas Técnicas de Soldadura CESOL, Octubre 2010.

[23] P. C. King, S. Zahiri, M. Jahedi y J. Friend, Surf. Coat. Tech. 205 (2010) 2.016-2.022.

[24] S. Gulizia, Clayton, A. Trentin, S. Vezzù, S. Rech, P. King, M. Jahedi y M. Guagliano, International Thermal Spray Conference \& Exposition 2010, Thermal Spray: Global Solutions for Future Application (DVS-ASM), Published: May 2010 pp. 80-83.

[25] K. Kim, M. Watanabe y S. Kuroda, Surf. Coat. Tech. 204 (2010) 2.175-2.180.

[26] S. H. Zahiri, C. I. Antonio y M. Jahedi, J. Mater. Process. Tech. 209 (2009) 922-929.
[27] C.K.S. Moy, J. Cairney, G. Ranzi, M. Jahedi y S. P. Ringer, Surf. Coat. Tech. 204 (2010) 3.739-3.749.

[28] T. Hussain, D.G. Mccartney, P. H. Shipway y T. Marrocco, International Thermal Spray Conference \& Exposition 2010, Thermal Spray: Global Solutions for Future Application (DVSASM), Published: May 2010 pp. 379-384.

[29] N. Cinca, M. Barbosa, S.Dosta y J.M. Guilemany, Surf. Coat. Tech. Volum 205(4) (2010) 1.0961.102 .

[30] S. Dosta, N. Cinca, M.Barbosa y J.M. Guilemany, International Thermal Spray Conference. ITSC 2010. Ref 1923.CD ROM. ISBN 978-3-87155590-9. ASM Thermal Spray Society. Singapore. (2010).

[31] D. Rafaja, T. Schucknecht, V. Klemm, A. Paul y H. Berek, Surf. Coat. Tech. 203 (2009) 3.2063.213.

[32] G. Bae, K. Kanga, J-J Kimb y C. Leea, Mater. Sci. Eng. A 527 (2010) 6.313-6.319.

[33] H-J. Choi, M. Lee y J.YouL Lee, Nucl. Eng. Des. 240 (2010) 2.714-2.720.

[34] B. Wielage, T. Grund, C. Rupprecht y S. Kuemmel, Surf. Coat. Tech. 205 (2010) 1.115-1.118.

[35] S. Marx, A. Paul, A. Kohler y G. Huttl, J. Therm. Spray. Tech. 15(2) June (2006) 177-183.

[36] S. Cadney, M. Brochu, P. Richer y B. Jodoin, Surf. Coat. Tech. 202 (2008) 2.801-2.806.

[37] K. Onizawa, J. Jansen, M. Schulze, F. Gärtner y T. Klassen, International Thermal Spray Conference \& Exposition 2010, Thermal Spray: Global Solutions for Future Application (DVSASM), Published: May 2010 pp. 163-166.

[38] H. Koivuluoto, G. Bolelli, L. Lusvarghi, F. Casadei y P. Vuoristo, Surf. Coat. Tech. 205 (2010) 1.103-1.107.

[39] R. Lupoi y W. O’Neill, Surf. Coat. Tech. 205 (2010) 2.167-2.173.

[40] Sova, D. Pervushin y I. Smurov, Surf. Coat. Tech. 205 (2010) 1.108-1.114.

[41] X.J. Ning, J.H. Jang, H.J. Kim, C.J. Li y C. Lee, Surf. Coat. Tech. 202 (2008) 1.681-1.687.

[42] C. Lorenzana, S. Dosta, M.Villa, J.Fernández y J. M. Guilemany, XII Congreso Nacional de Tratamientos Térmicos y de Superficie. ISBN 978-84-693-6946-3. CD ROM. TRATERMAT 2010. Pamplona. España. (2010).

[43] Q. Zhang, C.J.Li, C.X. Li, G.J. Yang y S.C. Lui, Surf. Coat. Tech. 202 (2008) 3.378-3.384.

[44] P. Richer, A. Zúñiga, M. Yandouzi y B. Jodoin, Surf. Coat. Tech. 203 (2008) 364-371.

[45] P. Richer, M. Yandouzi, L. Beauvais y B. Jodoin, Surf. Coat. Tech. 204 (2010) 3.962-3.974.

[46] Y. Li, C.-J. Li, Q. Zhang, L.-K. Xing y G.-J. Yang, International Thermal Spray Conference 
\& Exposition 2010, Thermal Spray: Global Solutions for Future Application (DVS-ASM), Published: May 2010 pp. 491-497.

[47] Y. Li, C.J. Li, G.J. Yang y L.K. Xing, Surf. Coat. Tech. 205 (2010) 2.225-2.233.

[48] N.Cinca, S.Dosta y J.M. Guilemany, Memorias del XI Congreso Nacional de Materiales. Vol 1. pp 245. ISBN 978-84-92522-24-8. Universidad de Zaragoza- Sociedad Española de Materiales. Zaragoza.(2010).

[49] S. Yoona, J. Kima, G. Baea, B. Kimb y C. Leea, J. Alloy. Compd. 509 (2011) 347.353.

[50] L. Ajdelsztajn, A. Zúñiga, B. Jodoin y E.J. Lavernia, Surf. Coat. Tech. 201 (2006) 2.109. 2.116.

[51] H. Lee, S. Lee y K. Ko, J. Mater. Process. Tech. 209 (2009) 937-943.

[52] K. Spencer y M.-X. Zhang, Surf. Coat. Tech. 203 20-21: 3.019-3.025.

[53] H. Lee, S. Lee, H. Shin y K. Ko, J. Alloy. Compd. 478 (2009) 636-641.

[54] S. Tria, O. Elkedim, W.Y. Li y H. Liao, J. Alloy. Compd. 483 (2009) 334-336.

[55] T. Ando, A. Miyazoe, N. Hirota, K. Tatamidani, H. Wada, S.Tsukamoto, O. Ozaki y T. Kiyoshi, Surf. Coat. Tech. 205 (2010) 1.990-1.994.

[56] W.Y. Li, G. Zhang, H.L. Liao y C. Coddet, J. Mater. Process. Tech. 202 (2008) 508-513.

[57] W.Y. Li, C. Zhang, H. Liao, J. Li y C. Coddet, Surf. Coat. Tech. 202 (2008) 4.855-4.860.

[58] Q. Wang, K. Spencer, N. Birbilis y M.X. Zhang, Surf. Coat. Tech. 205 (2010) 50-56.

[59] D. Poirier, J.-G. Legoux, R. A. L. Drew y R. Gauvin, J. Therm. Spray Tech. 20, Numbers 1-2, January (2011) 275-284(10).
[60] J.M. Miguel, J.M. Guilemany y S. Dosta, Surf. Coat. Tech. 205 (2010) 2.184-2.190.

[61] A. Siao Ming Ang, C, C. Berndt y P. Cheang, Surf. Coat. Tech. 205 (2011) 3.260-3.267.

[62] P.H. Gao, C.J. Li, G.J. Yang, Y.G. Li y C.X. Li, Surf. Coat. Tech. 203 (2008) 384-390.

[63] S. M. Ang, C. Berndt, P. Cheang y J. Wang, International Thermal Spray Conference \& Exposition 2010, Thermal Spray: Global Solutions for Future Application (DVS-ASM), Published: May 2010, pp. 768-773.

[64] H.J. Kima, C.H. Leeb y S.Y. Hwanga, Mater. Sci. Eng. A 391 (2005) 243-248.

[65] J.S. Kim, Y.S. Kwon, O.I. Lomovsky, D.V. Dudina, V.F. Kosarev, S.V. Klinkov, D.H. Kwon y I. Smurov, Compos. Sci. Technol. 67 (2007) 2.292-2.296.

[66] M. Yamada, H. Isago, K. Shima, H. Nakano y M. Fukumoto, International Thermal Spray Conference \& Exposition 2010, Thermal Spray: Global Solutions for Future Application (DVSASM), Published: May 2010, pp. 172-176.

[67] J.-O. Kliemann, H. Gutzmann, F.Gärtner, T.Klassen, I. Jursic, Braunschweig/d y C. Borchers, International Thermal Spray Conference \& Exposition 2010, Thermal Spray: Global Solutions for Future Application (DVSASM) Published: May 2010, pp. 90-95.

[68] F. Wang, D. Zhang, S. Zheng y B. Qi, Int. J. Hydrogen Energ. 35 (2010) 8.206-8.215.

[69] J. C. Lee, H. J. Kang, W. S. Chu y S. H. Ahn, CIRP Annals - Manufacturing Technology 56, Issue 1 (2007) 577-580.

[70] V. F. Kosarev, S. V. Klinkov y A. Sova, Recent Patents on Engineering 1(2007) 35-42. 